\title{
1 The DLR SAR Calibration Center
}

Jens Reimann, Marco Schwerdt, Björn Döring, Sebastian Raab, Daniel Rudolf,

4 Matthias Jirousek, Kersten Schmidt, Nuria Tous Ramon, John Mohan Walter Anton, Gabriel Adolfo Castellanos Alfonzo and Manfred Zink

\{Deutsches Zentrum für Luft- und Raumfahrt, Institut für Hochfrequenztechnik und Radarsysteme, Oberpfaffenhofen, Germany\}

Correspondence to: Jens Reimann (jens.reimann@dlr.de)

9

Abstract

A SAR sensor, like every other measurement device, has to be calibrated to perform quantitative measurements. During this process all essential parameters of a SAR image are linked to their geophysical quantities, like the pixel location, their complex-valued backscatter and polarimetric information. The Germany Aerospace Center (DLR) has been a key player in this field for more than 25 years and has concentrated its expertise and technology in performing the calibration of spaceborne SAR sensors to the DLR SAR Calibration Center at the Microwave and Radar Institute. During the last decades the DLR SAR Calibration Center has developed plenty of novel techniques and dedicated reference targets for spaceborne SAR system calibration. Some of these techniques which are also applied to current spaceborne SAR missions like Sentinel-1, TerraSAR-X and TanDEM-X will be presented along with the achievable performance.

\section{Introduction}

24 While the complexity of modern spaceborne SAR sensors increases, the calibration requirements become more and more stringent. We have to deal with highly agile antennas featuring a raising number of beams and sophisticated operation modes like TOPS (Terrain Observation by Progressive Scans, [1]) or sliding spotlight. Current SAR systems are operational sensors providing high quality products to scientific users and a growing commercial market. For such missions it is important to reduce the in-orbit commissioning 
1 phase to the bare minimum, which puts another tight constraint on the calibration activities.

2 These contracting demands require efficient calibration strategies individually adapted to the

3 specific needs of each SAR mission.

4 DLR's Microwaves and Radar Institute has gathered experience in the calibration of spaceborne SAR sensors for many years: starting with ERS-1 (1991) and SIR-C/X-SAR (1994), followed by SRTM (2000) as well as TerraSAR-X (2007), TanDEM-X (2010) and Sentinel-1A (2014) to name the most important ones. The outstanding results with TerraSAR$X \&$ TanDEM-X and several requests by ESA and other international partners triggered the initiative to establish the DLR SAR Calibration Center combining the expertise of an experienced team with adequate facilities and analysis tools. The latest mission supported by the SAR Calibration Center was Sentinel-1A for which in 2014 an independent commissioning phase was performed.

\section{DLR SAR Calibration Center}

The DLR SAR Calibration Center is a well-equipped facility for the calibration of spaceborne SAR systems. An important element of this facility is the infrastructure required to prepare and execute large calibration campaigns. It owns a multitude of calibration targets for different frequency bands and operates and maintains a large calibration field in Southern Germany (Figure 1). This calibration field consists of more than 30 sites hosting $1.5 \mathrm{~m}$ and $3 \mathrm{~m}$ trihedral corner reflectors.

Six new calibration sites have been added to this calibration field in 2014 for ESA's Sentinel-1 mission. Three of the sites have been equipped with remote-controlled trihedral corner reflectors (Figure 2), the three other sites host in-house developed C-band transponders (Figure 3) named 'Kalibri'.

Beside the transponder development team, the DLR SAR Calibration Center has experts for every step required during calibration which guarantees constant development in the appropriated fields of work. During the commissioning phase of a spaceborne SAR system all members of the calibration team work in parallel on the mission to minimized calibration time. 


\section{Calibration Targets}

2 A radar transponder is an active device which receives an electromagnetic wave, optionally 3 modifies it (e.g. amplification or time delay), and finally retransmits it into space. For

4 calibration purposes transponders with high amplification gain are used to improve the signalto-background ratio (SBR) over passive targets. They can be also built more flexible i.e. to produce different polarimetric signatures. Furthermore transponders can be extended to be usable as ground receivers recording the signals transmitted by the SAR satellite. Amongst others these data can further be used to retrieve the azimuth pattern of the SAR antenna or to analyze individual radar pulses.

The in-house developed C-band transponders 'Kalibri' follow a two-antenna design which enables small time delay between receive and transmit and large radar cross sections (RCS) due to reduced back coupling, see Figure 4. All transponder electronics including the antennas are place inside a water-proof housing. In combination with a precise temperature regulation within the box this modern design allows a very stable operation (Table 1). The transponder electronics is split into widely independent modules: the temperature controlling, digital data handling and recording and radio frequency parts are working autonomously and can be replaced individually. This does not only simplify maintenance, but also allows the adaption to other frequency bands: an additional X-band version of the 'Kalibri' transponder is likewise developed.

The absolute radiometric gain calibration of a target like a transponder is a critical task as it defines the lower boundary for the overall radiometric accuracy of the whole SAR system. The calibration for the 'Kalibri' transponders was performed using different measurement setups [2] to provide additional confidence in the results of this extremely critical parameter: Comparison measurements with precisely known trihedral corner reflectors and a circular metal plate have been performed in the DLR's own compact test range. Secondly, the transponders were used as a radar, measuring trihedral corner reflectors in far field regime. In a third setup, transponders and several corner reflectors were placed within one scene of the Canadian C-band SAR satellite RADARSAT-2 to retrieve the RCS of the transponders without relying on the original satellite calibration [3]. The detailed analysis of the whole calibration campaign can be found in [4].

31 As part of the transponder calibration efforts a novel calibration technique was developed. 32 Extending the well-known 'Three Antenna Method' for the absolute gain determination of 
1 antennas, the 'Three Transponder Method' was proposed [5]. This technique allows the

2 retrieval of the RCS of transponders without using a dedicated RCS reference. Instead

3 distance and frequency measurements are performed which can be conducted with high

4 precision. Due to the measured quantities, this technique allows an easy trace back of the RCS measurement to the International System of Units with known uncertainty.

6 For calibration of ESA's Sentinel-1 mission a second type of reference targets was also employed: three novel $2.8 \mathrm{~m}$ trihedral corner reflectors were installed in DLR's calibration field. They are very precisely manufactured with a surface accuracy of better than $1 \mathrm{~mm}$ which was verified on-site by dedicated measurements. This precision guarantees only small deviation of the corner reflector's RCS from its theoretical value (Table 2).

11 These new corner reflectors can be remotely controlled enabling unique features. The drives of the corner reflector axes allow a very accurate alignment of better than $0.01 \mathrm{deg}$ which improves the overall accuracy and result in a constant phase center for a given alignment. This is an important feature for radar interferometry. To protect the inner part from hazardous weather conditions and dirt the corner reflectors are moved to a so called 'parking position' facing downwards when not used. Furthermore remote controlling of the corner reflectors enables the DLR SAR Calibration Center to offer these targets to other agencies for calibration.

\section{Calibration Procedures}

Every SAR mission requires an individual calibration strategy to be developed to satisfy its system requirements and to release SAR products as soon as possible. One already proven and established concept for calibration is based on an antenna model approach [6] to predict the performance of the hundredths of beams of a modern active phased array antenna, which is often employed. The calibration itself then consists of:

- Internal Calibration, for stable instrument performance over time.

- Geometric Calibration, to assign the SAR data to the geographic location on the Earth's surface.

- Antenna Pointing Determination, to precisely retrieve the beam direction of the antenna. 
- Antenna Model Verification, to verify the precision of the reference patterns generated by the model, including the gain offset between different beams.

- Polarimetric Calibration to align data between different polarimetric channels.

- Absolute Radiometric Calibration, for radiometric bias correction of SAR data products.

\section{Calibration Techniques}

Absolute radiometric calibration is one of the most critical tasks performed during commissioning phase of a SAR satellite. Several reference targets have to be used to achieve today's accuracy demands. The existing and well-equipped calibration field of the DLR SAR Calibration Center is a big advantage when performing this task.

The impulse response from each aligned reference target can be utilized to derive the calibration factor of the SAR instrument. An increasing number of targets or overpasses can be used to reduce the statistical uncertainty associated with every single measurement. For the retrieval the complex-valued image is interpolated in frequency domain, the total target power is accumulated and finally compensated for noise. This value will then be compared to the RCS of the target to derive the calibration factor. Linking all uncertainties, i.e. from the RCS measurement, the target's RCS knowledge and the propagation path a combined uncertainty for the measurement can be derived.

The most important point with respect to radiometric accuracy is the stability of a system like TerraSAR-X. Due to a re-calibration campaign two years after launch, extended by measuring several beams and a couple of reference targets, a precise evaluation of the stability with a high confidence level could be achieved. For this purpose, the absolute calibration factor averaged over all measurements performed in 2009, see Figure 5, has been compared to that derived from measurements performed during the commissioning phase in 2007. Consequently, an offset between both values is a measure for the stability. For a period of two years a radiometric stability of $0.15 \mathrm{~dB}$ could be proved for TerraSAR-X. This stability is more than one magnitude better than the requirement with $0.5 \mathrm{~dB}$ over six months.

For the TanDEM-X system an absolute radiometric accuracy of $0.14 \mathrm{~dB}$ was achieved for StripMap operation during the commissioning phase. Considering furthermore the same 31 radiometric stability as the earlier launched TerraSAR-X satellite and propagation 
1 uncertainties of $0.25 \mathrm{~dB}$, an absolute radiometric accuracy of less than $0.5 \mathrm{~dB}$ could be

2 achieved for the mission time [6].

3 Linking a pixel position of the SAR image to a location on Earth is called geometric 4 calibration. Beside a precise knowledge of the target and the satellite location, the propagation path has to be known. Especially, the delay, bending and dispersion of the atmosphere, namely troposphere and ionosphere, have to be considered [7], [8].

7 The influence of the troposphere on the signal is frequency-independent and can be split into a slowly variating hydrostatic (several meters in range) and a weather-dependent wet delay (few centimeters offset). The ionosphere has a frequency dependency and is dispersive, which mostly affects signals with frequencies below S-band. The range shift $\Delta r$ can be quantified by the total electron count (TEC) on the propagation path [9]:

$$
\Delta \mathrm{r} \approx \frac{40.31 \frac{m^{2}}{s^{2}}}{f^{2}} \cdot T E C
$$

where $\mathrm{f}$ is the frequency. This equation is also widely used by global navigation satellite systems (GNSS) like GPS or Galileo.

Faraday rotation is a second effect caused by the ionosphere. It depends not only on TEC, but also on the strength of the Earth's magnetic field along the propagation path:

$$
\psi=2.610^{-13} \cdot T E C \cdot B \cdot \lambda^{2} \cdot \cos (\theta)
$$

with $\psi$ in radian, TEC in electrons per $\mathrm{m}^{2}$, B the Earth's magnetic field, $\lambda$ the wavelength and $\theta$ the angle between the radar illumination and the magnetic field. The Faraday rotation angle $\psi$ is changing the orientation of linear polarized waves or expresses itself as an additional phase offset for circular polarization.

The accurate mapping of a SAR image pixel to the Earth surface is finally performed by using point targets like corner reflectors with known phase center as reference and accounting for propagation effects. The results from the TerraSAR-X mission are shown in Figure 6. For look angles up to about $40 \mathrm{deg}$, the slant range with a distance of about $600 \mathrm{~km}$ could be derived down to an accuracy of $6 \mathrm{~cm}$. At higher look angles, the variance of the residual error increases as the propagation path through the atmosphere is longer. Nevertheless, considering the complete range of look angles a pixel localization accuracy of $30 \mathrm{~cm}$ could be achieved for TerraSAR-X. 
1 To properly illuminate the scene and to correctly apply the antenna pattern correction during

2 SAR data processing the antenna pointing of a SAR instrument has to be known precisely.

3 The antenna pointing calibration estimates the difference between the real antenna pointing

4 and the desired one. This can be done by commanding regular beams or so called notch beams on the phase array antenna either in elevation or in azimuth. Based on the gain drop of notch beams, the boresight direction can accurately be recognized. For regular beams the maximum power has to be determined.

Scenes over the Amazon rainforest which have a very homogeneous backscatter characteristic can be used to derive the pointing in elevation. The azimuth or flight-direction pointing can be found by analyzing ground receiver recordings. Ground receivers are used to store the power received from the SAR instrument annotated with a precise time stamp. Knowing the satellite and target position, the azimuth antenna pattern and consequently the real pointing in flightdirection can be reconstructed from that data [15].

For TerraSAR-X and TanDEM-X the antenna pointing knowledge was proven to be better than $0.002 \mathrm{deg}$ in elevation, see Figure 7. By readjusting the attitude of the satellite, the detected mispointing of the antenna beam can still be compensated.

Recordings from ground receivers can also be used to verify the antenna model of the SAR instrument for different beams [13]. The modelled antenna pattern is compared to the reconstructed data from ground measurements. Even for the complicated case of TOPS (Terrain Observation with Progressive Scan) acquisition the DLR SAR Calibration Center was able to successfully check the antenna model of Sentinel-1A see [10], [11], and Figure 8. In TOPS operation hundredths of beams are commanded to steer the antenna main lobe from sub-swath to sub-swath over an area of interest. This can be also seen in Figure 8 were several main beams are visible (e.g. at about $-0.2 \mathrm{~s}, 0.1 \mathrm{~s}$ or $0.3 \mathrm{~s}$ ) together with the discontinuities in between which occur when switching to another sub-swath (e.g. at about 0.2 deg in azimuth). The DLR SAR Calibration Center is also permanently developing and improving its calibration techniques. The pulse coded calibration (PCC) method [12] is one of them. It can be applied to characterize the transmit-receive-modules (TRMs) of active phased array antennas. As part of the internal calibration of a SAR instrument, several measurements are performed on the phase array antenna where the phase shifters of the TRMs are varied 31 between 0 and 180 degree in a pseudo noise manner. From these measurements the deviation 32 of the TRMs from their nominal state can be retrieved while all modules are in full operation. 
1 The accuracy of this technique was proven to be better than some hundredth of a dB in amplitude and several tenth of a degree in phase [10].

Figure 9 shows the amplitude and phase drift of the Sentinel-1A instrument for a 25 minutes Stripmap data take (DT), and the corresponding temperature variation in the front-end. The instrument amplitude drift for such a long data take is less than $0.3 \mathrm{~dB}$, while the phase drifts about $12 \mathrm{deg}$. During this time, the front-end temperature increases by $16{ }^{\circ} \mathrm{C}$. Theoretically, this corresponds to an instrument drift of $0.012 \mathrm{~dB}$ and $0.007 \mathrm{deg}$ per minute, which proves the very high stability of the Sentinel-1A instrument and the accuracy of the PCC technique. Furthermore, the measured instrument drift is compensated for at raw data level during SAR data processing, so that the remaining instrument contribution to the SAR image is smaller than $0.1 \mathrm{~dB}$ in amplitude and only a few degrees in phase.

A wide variety of analysis and calibration tools have also been developed by the Microwave and Radar Institute during the last years. They support the calibration team in deriving the various calibration parameters during the commissioning phase and in nominal operation of a spaceborne SAR system. These tools are constantly enhanced and adapted to the individual SAR missions to meet their specific requirements.

The tool CALIX is used to perform point and distributed target analysis. It reads focused SAR data to extract image information like pixel location, resolution, side lobe level, background noise and absolute target energy for single point targets. Also polarimetric information can be extracted. The CALIX program can be used via a graphical user interface or by external scripts for semi-automatic analysis. But also analysis tools for internal calibration and for characterizing the antenna in-flight have been developed and are permanently improved and adapted for the corresponding mission. Other tools are dedicated to calculate the alignment angles for targets in the calibration field for a given satellite overpass or to design a calibration field based on the orbit of a future SAR satellite.

\section{Conclusion}

The complexity of SAR instruments and their calibration has increased during the last years while the demand on highly accurate spaceborne SAR data products rose. To keep pace with this development, the DLR SAR Calibration Center of the Microwave and Radar Institute has built up, maintained and extended its calibration facilities. Efficient calibration concepts have 
1 been developed based on innovative methods, like the antenna model approach or the PCC

2 technique. New innovative targets like remote-controlled trihedral corner reflectors or the 3 in-house developed C-band transponders 'Kalibri', as well as novel tools for product quality 4 control and performance analysis have been developed. An overview over the achievable 5 accuracies was given for the most relevant aspects of spaceborne SAR calibration 6 (summarized in Table 3).

7 


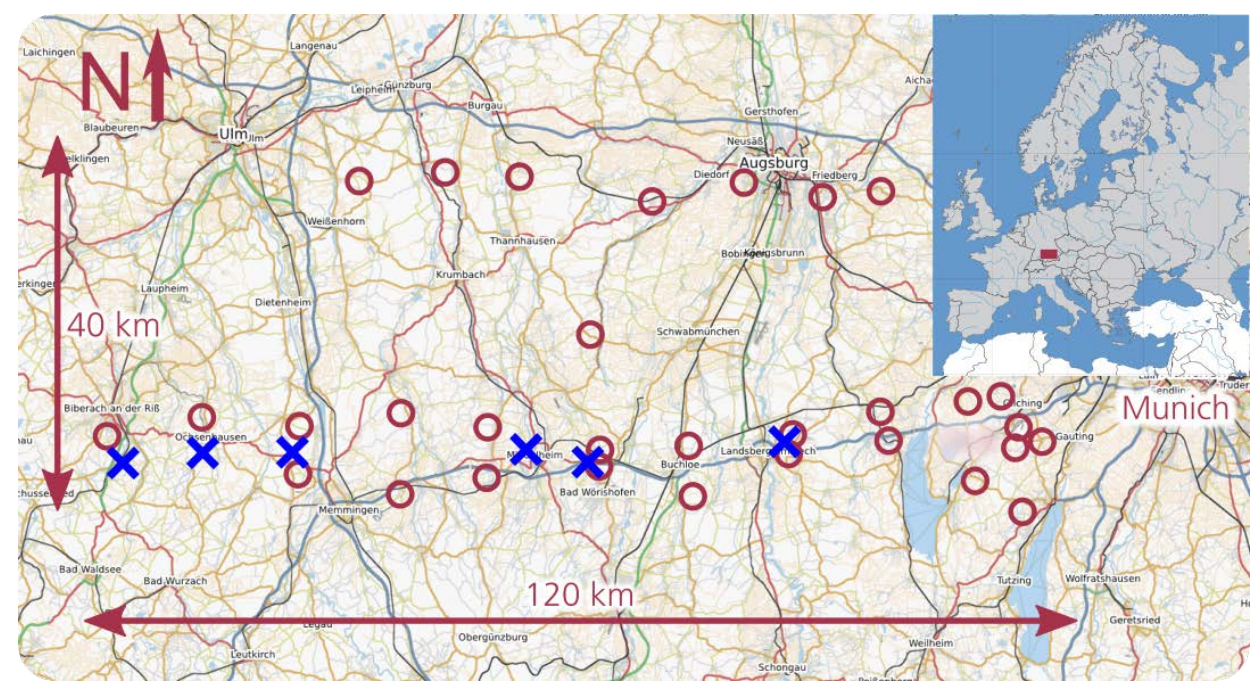

3 Figure 1 The DLR calibration field consists of 36 target positions with 20 corner reflectors 4 permanently installed. The newly installed six remote controlled targets are marked by blue crosses. (Map: (C) OpenStreetMap contributors, Overview: David Liuzzo, CC-BY-SA-2.0-de)

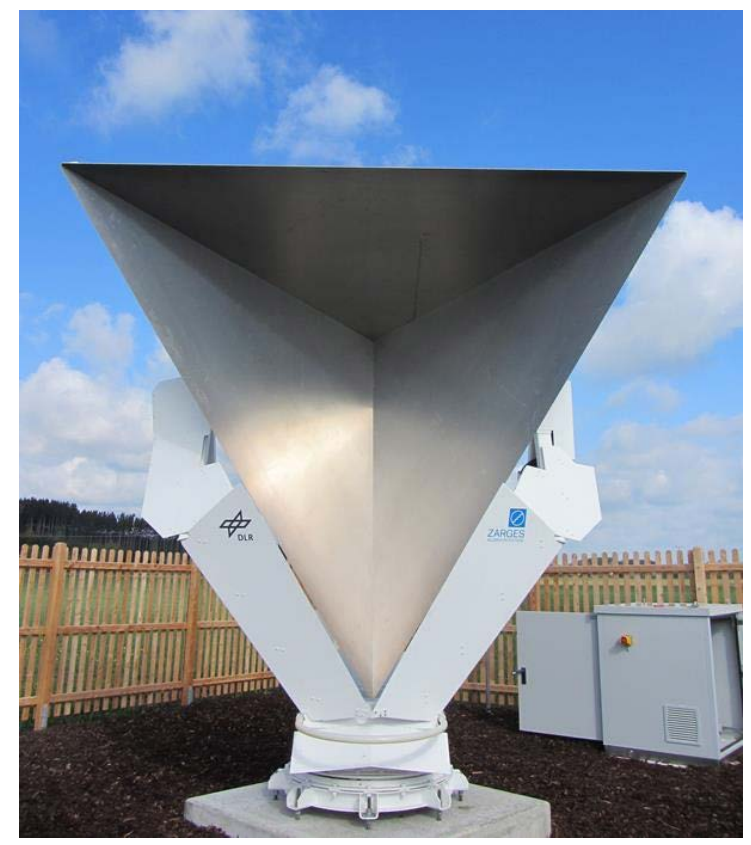

8 Figure 2 New remote-controlled corner reflector with a leg length of $2.8 \mathrm{~m}$. 


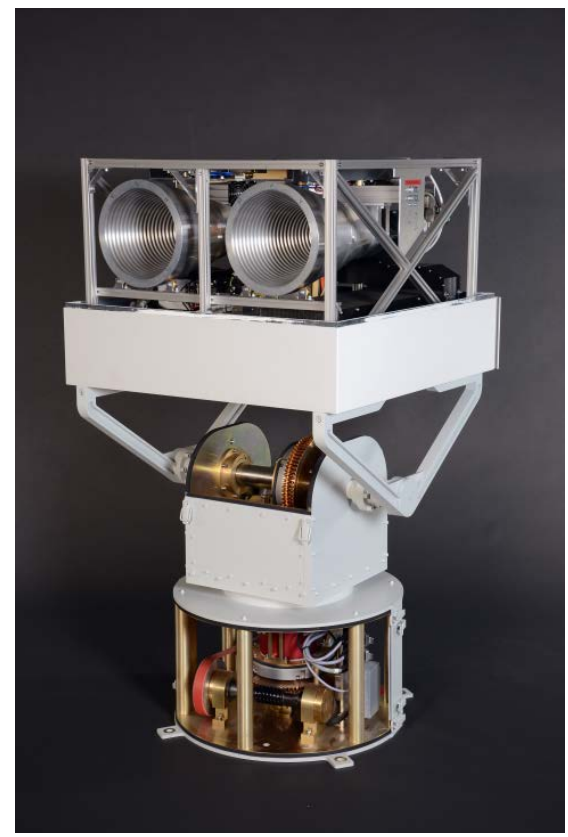

2 Figure $3 \mathrm{C}$-band remote-controlled 'Kalibri' transponder, housing of the positioner removed.

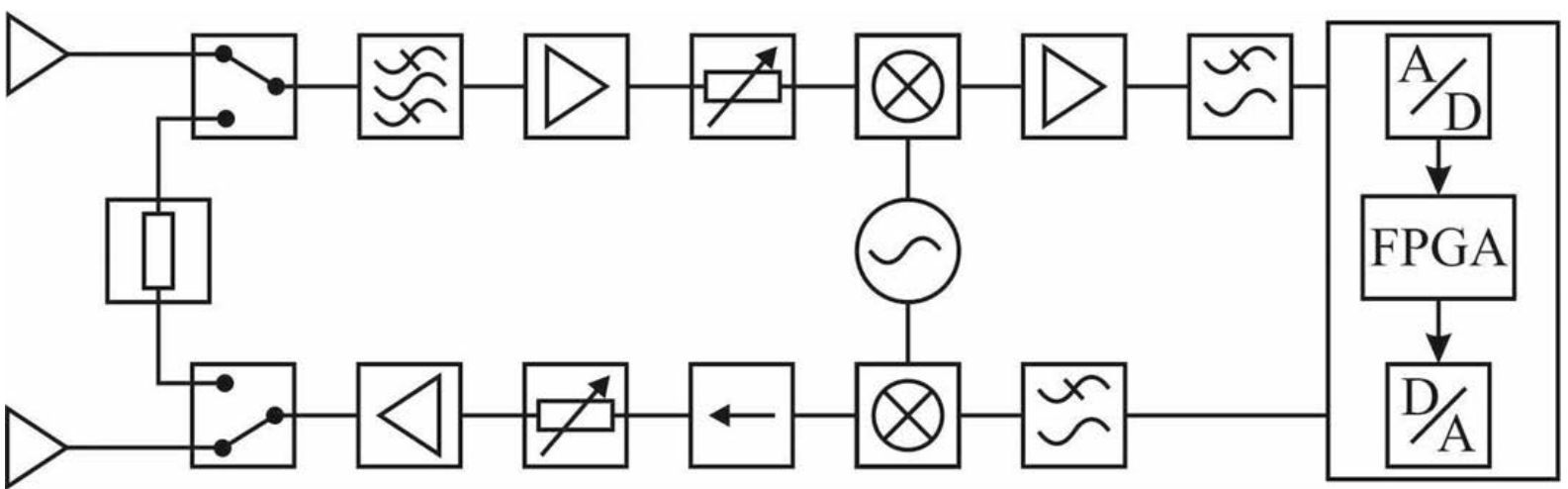

3 Figure 4 Basic scheme of the 'Kalibri' transponder including up- and down-conversion 5 stages. 


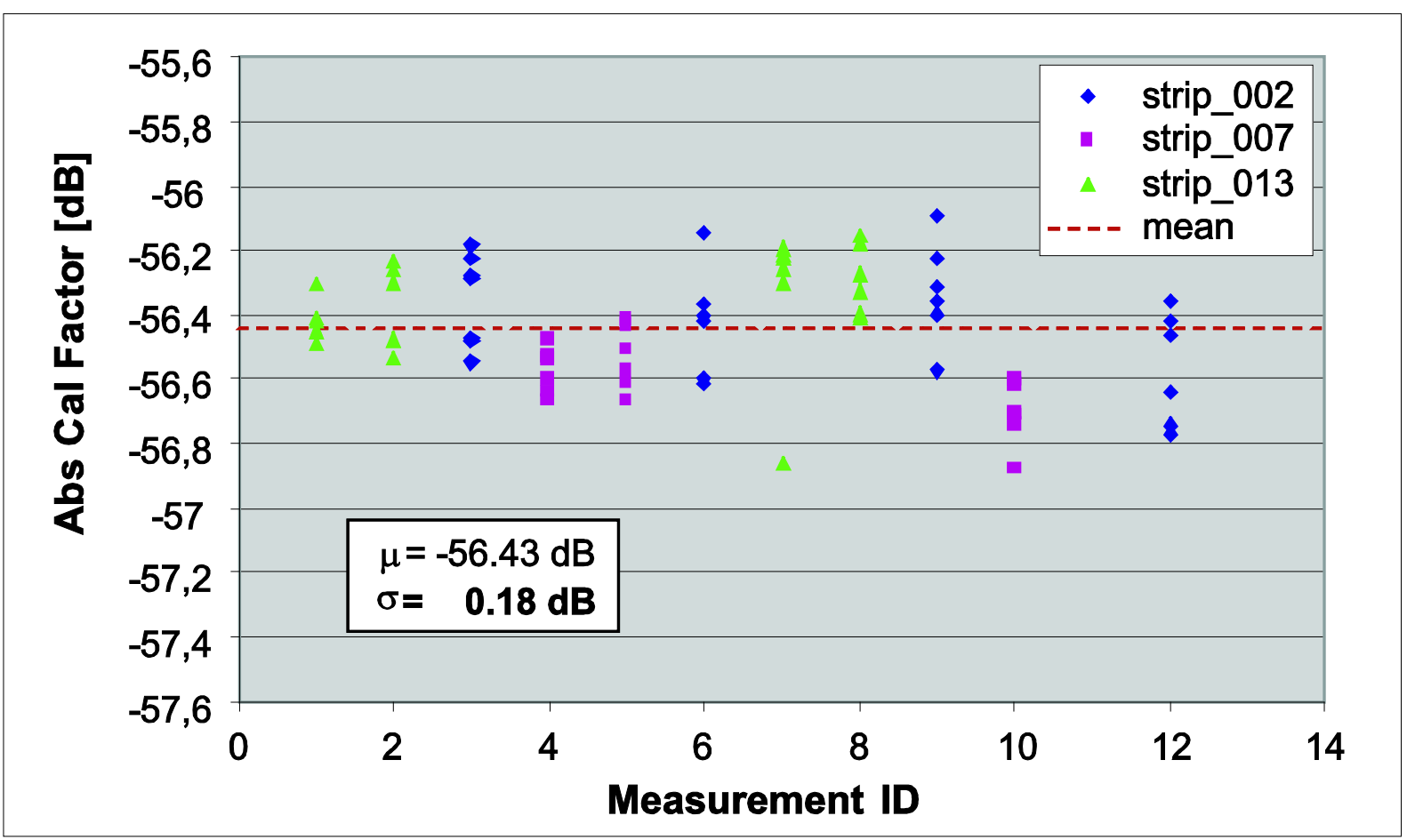

2 Figure 5 Absolute calibration factor versus measurement ID, derived from all reference 3 targets deployed for re-calibrating TerraSAR-X in 2009. The instrument was operated in 4 different StripMap beams [14].

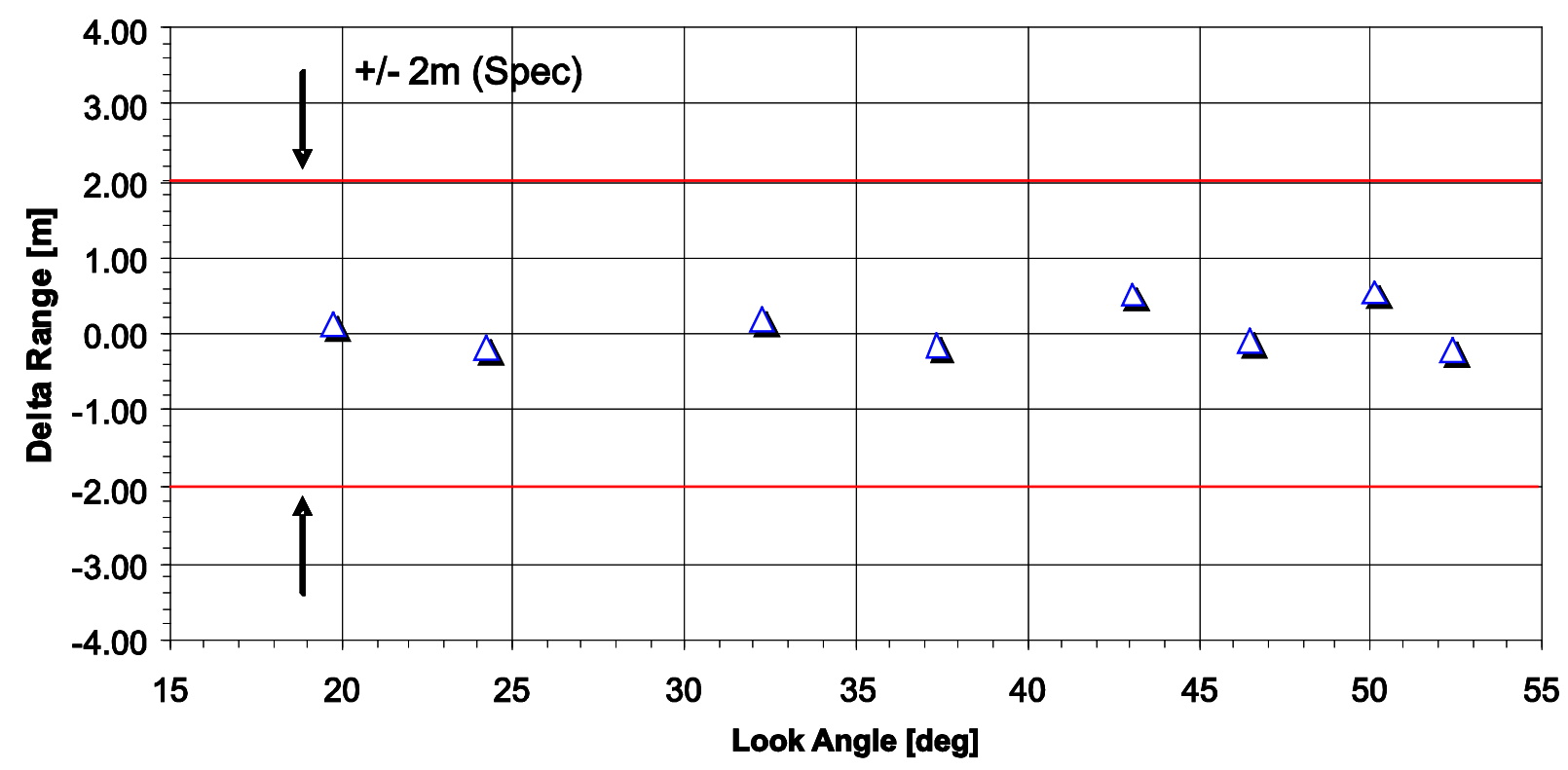

6 Figure 6 Residual slant range offset in dependence of the look angle after correcting for 7 instrument delay and propagation effects [6]. 


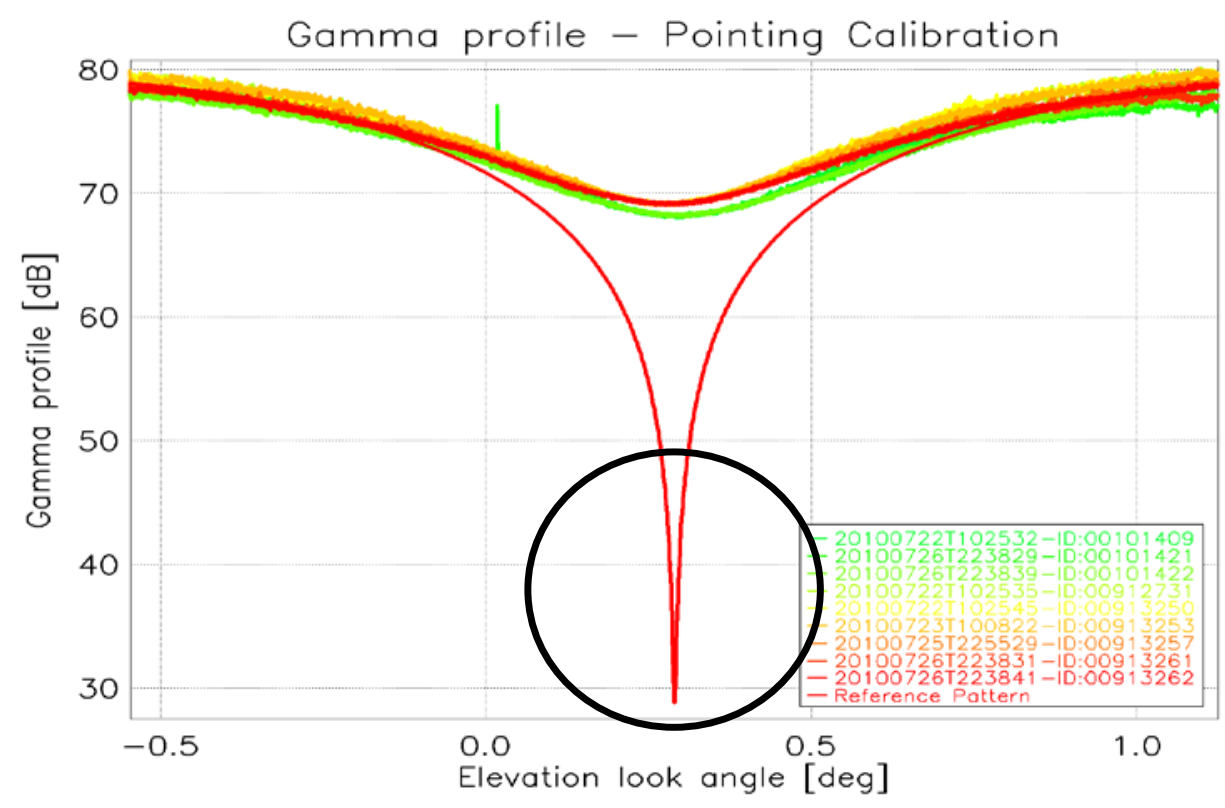

1

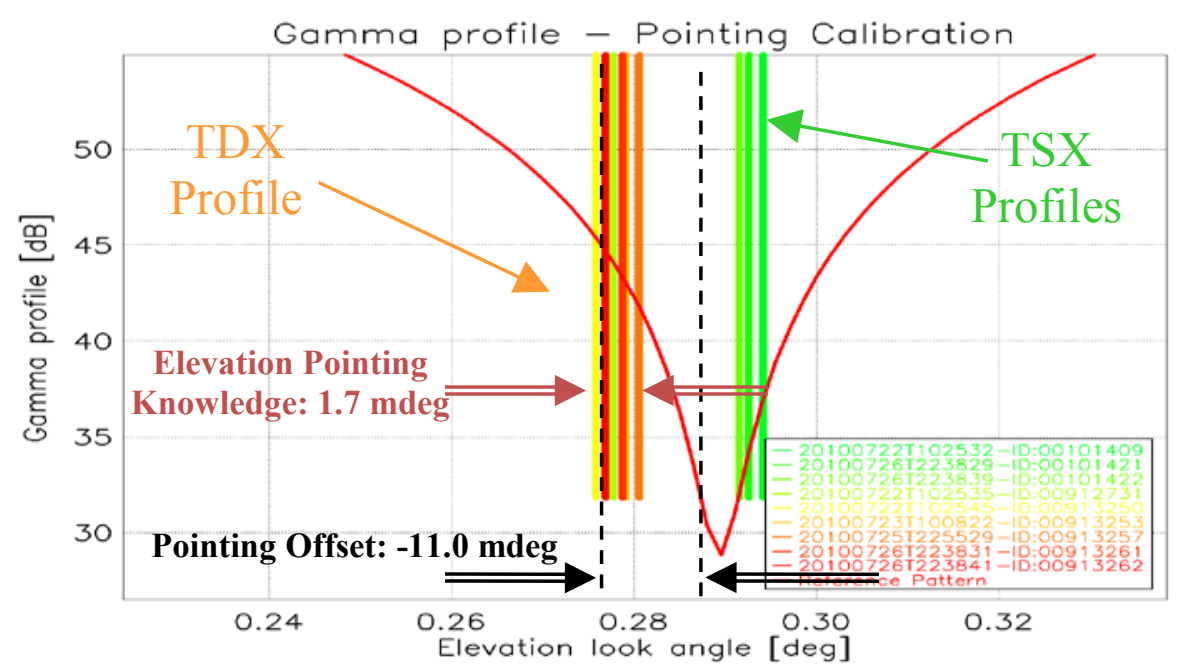

2

3 Figure 7 Beam pointing in elevation of TerraSAR-X determined by notch patterns measured 4 across the rainforest (gamma profile). The measurements depicted were performed at different 5 days. 


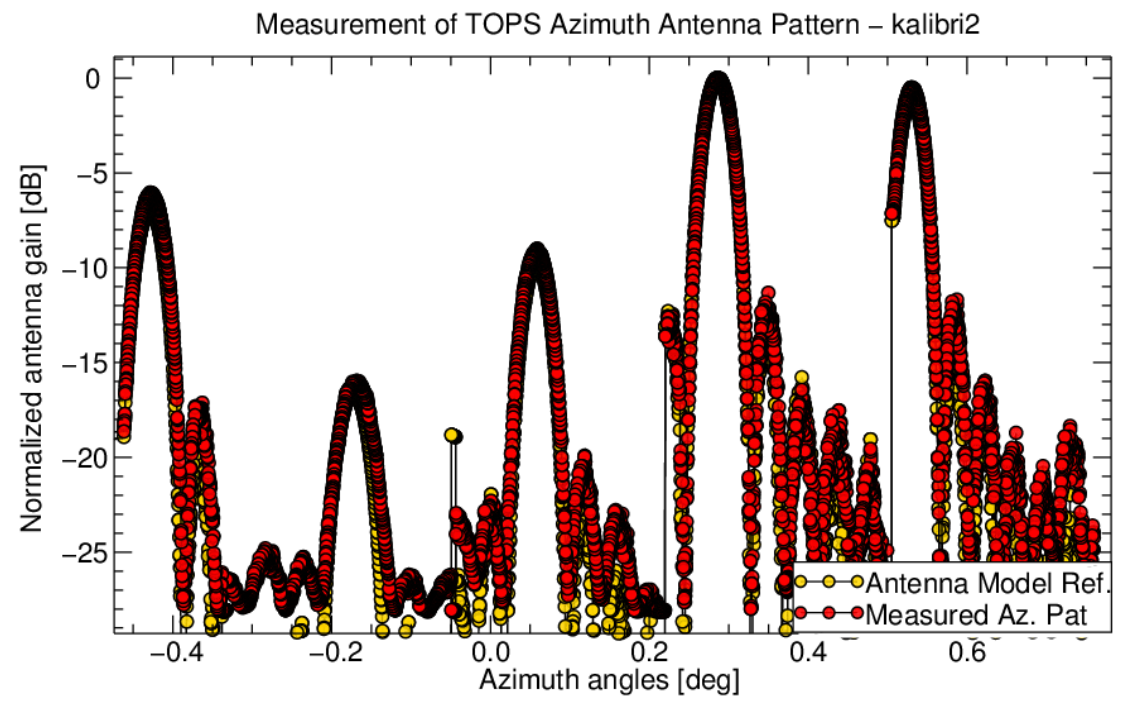

1

2 Figure 8 Comparison between antenna model and transponder measurements of an azimuth 3 pattern of the extrawide swath mode realized by TOPS operation of Sentinel-1A [11]. 

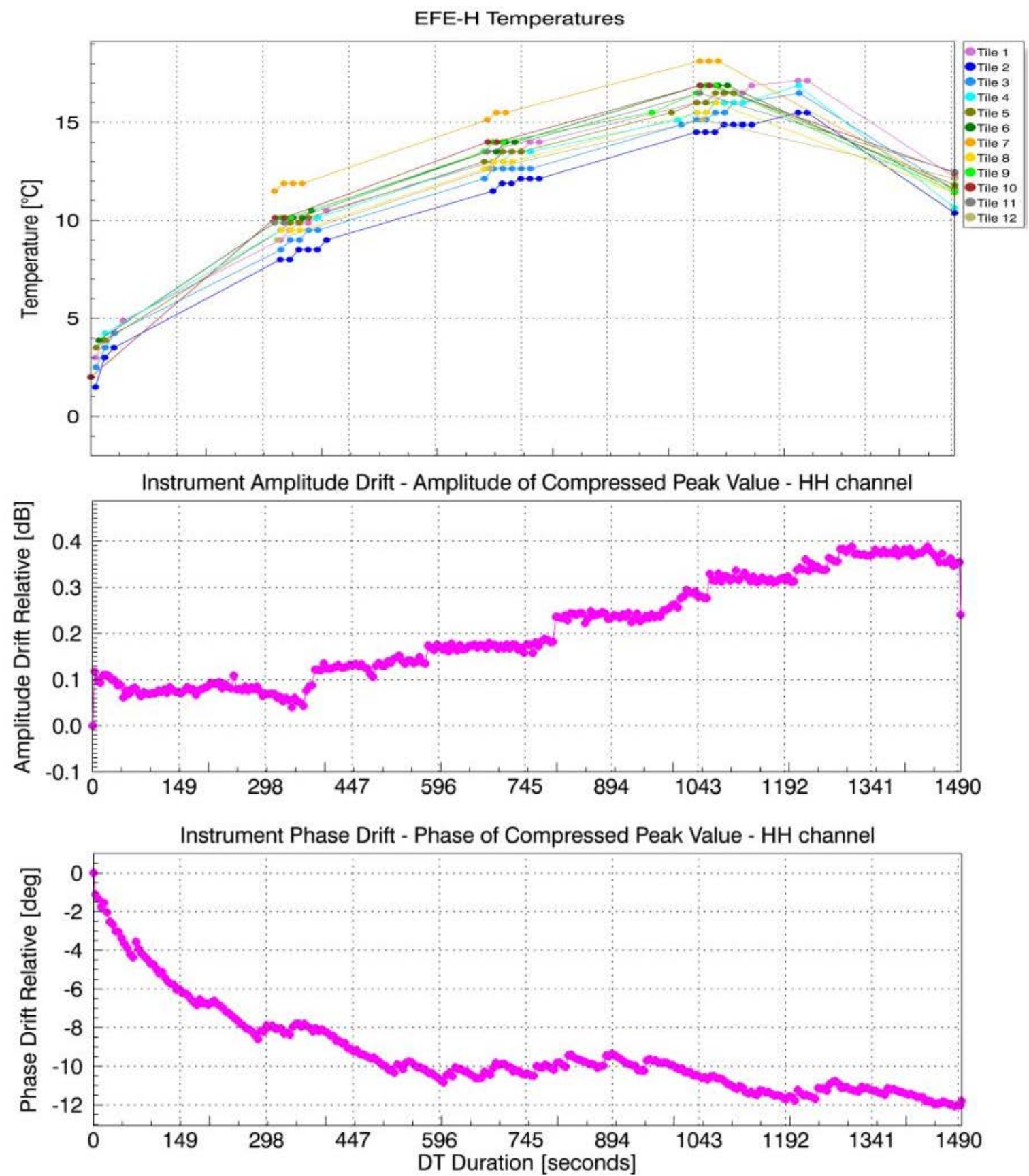

2 Figure 9 Front-end temperature drift (upper plot), instrument drift in amplitude (middle plot)

3 and instrument drift in phase (lower plot) of the co-polar channel ( $\mathrm{HH})$ for a 25 minutes long

4 Stripmap data take of Sentinel-1A acquired on the 06.08.2014 [10]. 
1 Table 1 Specification of the 'Kalibri' transponders in C-band

Center Frequency $\quad 5.405 \mathrm{GHz}$

Bandwidth $\quad 100 \mathrm{MHz}$

RCS $\quad 60 \mathrm{dBm}^{2}$

Radiometric Stability $\leq 0.1 \mathrm{~dB}(1 \sigma)$

Abs. Radiometric Accuracy $0.2 \mathrm{~dB}(1 \sigma)$

Polarization Adjustable backscatter

matrix

2

3 Table 2 Specification of the remote-controlled corner reflectors

Leg Length $\quad 2.8 \mathrm{~m}$

RCS (X-,C-,L-Band) $\quad 54.3,49.2,36.6 \mathrm{dBm}^{2}$

Mechanical Tolerance $\quad \leq 1.0 \mathrm{~mm}$

Abs. Radiometric Accuracy $0.2 \mathrm{~dB}(1 \sigma)$

Pointing Accuracy $\quad<0.1 \mathrm{deg}$ 
2 Table 3 Accuracy of the DLR SAR Calibration Techniques

\section{TRM Characterization}

Amplitude / Phase

$$
<0.2 \mathrm{~dB} /<2 \mathrm{deg}
$$

\section{Geometric Calibration}

Azimuth / Range

$$
\sim 10 \mathrm{~cm} / \sim 10 \mathrm{~cm}
$$

\section{Pointing Determination}

Azimuth / Elevation

$$
<2 \text { mdeg / }<2 \text { mdeg }
$$

Antenna Model Verification

Gain

$$
< \pm 0.2 \mathrm{~dB}
$$

\section{Radiometric Calibration}

Accuracy

Stability

$$
\begin{aligned}
& <0.3 \mathrm{~dB} \\
& <0.2 \mathrm{~dB}
\end{aligned}
$$




\section{References}

[1] F. De Zan, F. and A. Monti Guarnieri, "TOPSAR: Terrain Observation by Progressive Scans", IEEE Transactions on Geoscience and Remote Sensing, Vol. 44, No. 9, September 2006, pp 2352-2360.

[2] D. Rudolf, et al., "Absolute Radiometric Calibration of C-Band Transponders with Proven Plausibility," Proceedings of the European Conference on Synthetic Aperture Radar, 1-4, 2014

[3] B. J. Döring, K. Schmidt, M. Jirousek, R. Daniel, J. Reimann, S. Raab, A. John, and M. Schwerdt, "Hierarchical Bayesian data analysis in radiometric SAR system calibration: A case study on transponder calibration with RADARSAT-2 data," Remote Sensing, vol. 5, no. 12, pp. 6667-6690, 2013.

[4] S. Raab, et al., "Comparison of absolute radiometric transponder calibration strategies," Proceedings of the European Conference on Synthetic Aperture Radar, 1-4, 2014

[5] B. J. Döring, M. Jirousek, D. Rudolf, S. Raab, J. Reimann, and M. Schwerdt, "The three-transponder method: A novel method for accurate transponder RCS calibration," Progress in Electromagnetics Research (B), 2015.

[6] M. Schwerdt, B. Bräutigam, M. Bachmann, B. Döring, D. Schrank, and J. H. 9 Gonzalez, "Final TerraSAR-X Calibration Results Based on Novel Efficient Methods," in IEEE Transaction on Geoscience and Remote Sensing, 2010, Vol. 148, no.2.

[7] M. Jehle, D. Perler, D. Small, A. Schubert, and E. Meier, "Estimation of 3 atmospheric path delays in TerraSAR-X data using models vs. measurements," 4 Sensors, vol. 8, no. 12, pp. 8479-8491, 2008.

[8] O. Frey, E. Meier, D. Nüesch, and A. Roth, "Geometric error budget analysis for 6 TerraSAR-X," in 5th European Conference on Synthetic Aperture Radar EUSAR, 2004 , pp. 513-516.

[9] K. Davies, "Ionosphere Radio", IEEE Electromagnetic Waves Series 31, 1989, 9 Peter Peregrinus Ltd, Boulder, Colorado, USA. [10]M. Schwerdt, K. Schmidt, N. T. Ramon, G. C. Alfonzo, B. Döring, M. Zink, and P. 1 Prats, "Independent Verification of the Sentinel-1A System Calibration," in IEEE 32 Journal of Selected Topics in Applied Earth Observations and Remote Sensing 
1 (JSTARS), Special Issue on Synthetic Aperture Radar (SAR) New Techniques, 2 Missions and Applications, accepted, 2015.

3 [11]G. Castellanos Alfonzo, M. Schwerdt, S. Wollstadt, M. Bachmann, B. Döring, and

D. Geudtner, "First TerraSAR-X TOPS mode antenna pattern measurements using ground receivers" Int. J. Antennas Propag., vol. 2013, 7 p., 2013

[12]D. Hounam, M. Schwerdt, and M. Zink, "Active Antenna Module Characterisation by Pseudo-Noise Gating," in 25th ESA Antenna Workshop on Satellite Antenna Technology, Noordwijk, Netherlands, 2002.

[13]M. Schwerdt, D. Schrank, M. Bachmann, J. H. Gonzalez, B. Döring, N. TousRamon, and J. W. Antony, "Calibration of the TerraSAR-X and the TanDEM-X 1 Satellite for the TerraSAR-X Mission," in 9th European Conference on Synthetic 2 Aperture Radar, 2012.

3 [14]Schwerdt, M.; Gonzalez, J.H.; Bachmann, M.; Schrank, D.; Schulz, C.; Döring, B., 4 "Monostatic calibration of both TanDEM-X satellites," in Geoscience and Remote 5 Sensing Symposium (IGARSS), 2010 IEEE International, vol., no., pp.2636-2639, 25-30 July 2010

1 [15]K. Schmidt, M. Schwerdt, N. Tous Ramon, G. Castellanos Alfonzo,

8 and M. Zink "Sentinel-1A System Calibration using innovative Point Targets" Wave 9 Propagation in Communication, Microwaves Systems and Navigation (WFMN), 2015-02-11 - 2015-02-13, Chemnitz, Germany. 